\title{
Germline deletion of chromosome 2p16-21 associated with Lynch syndrome
}

\author{
Soichiro Natsume ${ }^{1}$, Tatsuro Yamaguchi ${ }^{1,2}$, Hidetaka Eguchi ${ }^{3}$, Yasushi Okazaki $\mathbb{1}^{3}$, Shin-ichiro Horiguchi ${ }^{4}$ and \\ Hideyuki Ishida ${ }^{5}$
}

\begin{abstract}
We identified a Japanese patient with Lynch syndrome with a novel large germline deletion of chromosome 2p16-21, including the EPCAM, MSH2, and KCNK12 genes. The proband was a 46-year-old man with ascending colon cancer. The clinical significance of germline KCNK12 gene deletion, which encodes one of the subfamilies of two-pore-domain potassium channels, is still unknown.
\end{abstract}

Lynch syndrome (LS) is an autosomal dominant inherited disorder caused by a germline pathogenic variant in mismatch repair (MMR) genes, including the $M L H 1, M S H 2, M S H 6$, and PMS2 genes. Inactivation of the MMR gene, which is caused by both germline and/or somatic variants, leads to an increased frequency of replication errors in repeated sequences in the coding regions of cancer-related genes, resulting in the development of LS-associated tumors ${ }^{1}$. Therefore, LS is characterized by the development of various cancers at a young age, including colorectal cancer, endometrial cancer, small bowel cancer, and urinary tract cancer.

The EPCAM gene, which is located $17 \mathrm{~kb}$ upstream of $M S H 2$, is not an MMR gene. However, germline deletion of the 3' end of the EPCAM gene causes LS because EPCAM deletion leads to $M S H 2$ gene silencing by promoter hypermethylation and $M S H 2$ inactivation ${ }^{2}$. The proportion of EPCAM gene deletions in LS is estimated to be $1.0-3.0 \%^{3,4}$, and to date, only a few families have been reported in $\operatorname{Japan}^{5}$.

Here, we identified a Japanese patient with LS with a novel large germline deletion of chromosome 2p16-21,

Correspondence: Tatsuro Yamaguchi (tatsuro@yamaguchi.email.ne.jp)

${ }^{1}$ Department of Surgery, Tokyo Metropolitan Cancer and Infectious Diseases Center Komagome Hospital, Tokyo, Japan

2Department of Clinical Genetics, Tokyo Metropolitan Cancer and Infectious Diseases Center Komagome Hospital, Tokyo, Japan

Full list of author information is available at the end of the article including the EPCAM, MSH2, KCNK12 genes. This novel finding provides evidence of germline deletion in this region and raises questions regarding the deletion of the KCNK12 gene.

The proband was a 46-year-old man who was referred to our hospital with abdominal pain. A colonoscopy demonstrated an ascending colonic tumor, and biopsy revealed adenocarcinoma in the ascending colon. However, computed tomography showed no evidence of lymph node metastasis, distant metastasis, or other organ diseases. In addition, his mother had been treated for colon cancer, glioblastoma, gastric cancer, and endometrial cancer, and she died from glioblastoma at the age of 67 years old (Fig. 1).

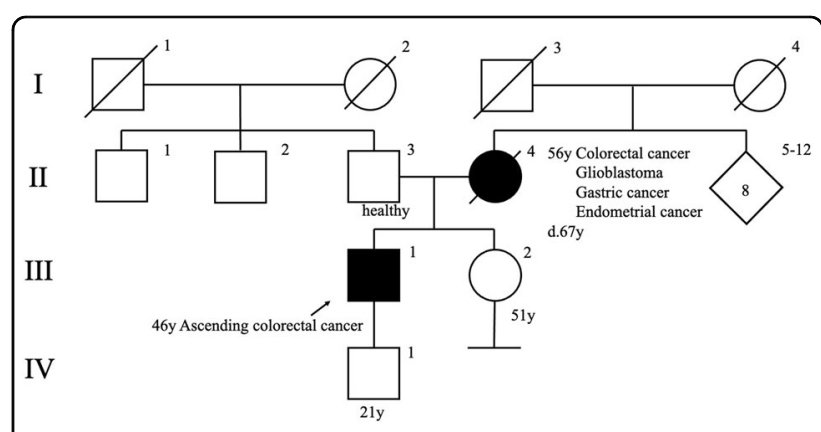

Fig. 1 Pedigree of the patients. The arrow indicates the proband. A filled symbol indicates a person with colorectal cancer. 


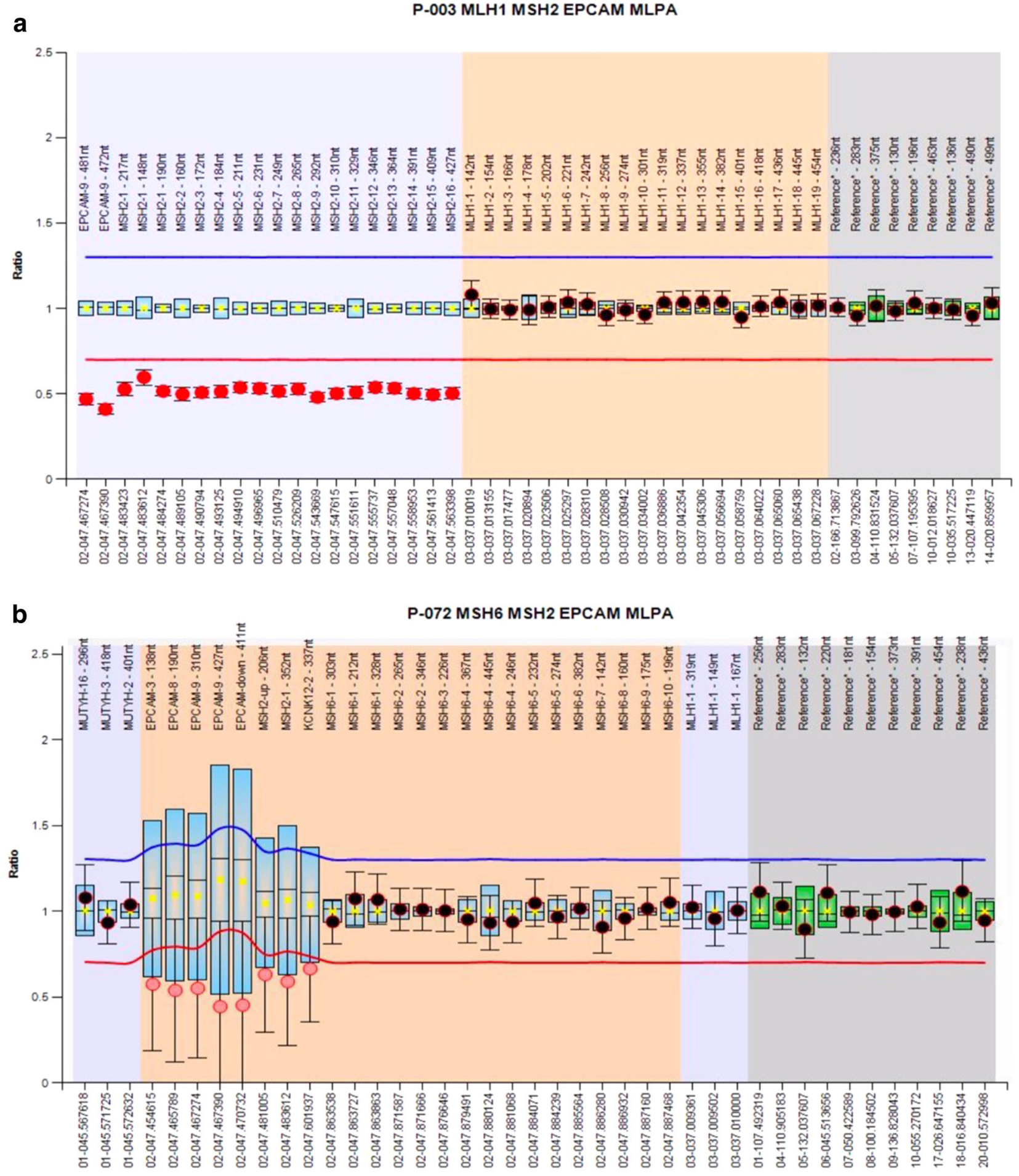

Fig. 2 Multiplex ligation-dependent probe amplification (MLPA) analysis of mismatch repair genes. Ratio chart for MLPA analysis Using SALSA MLPA Probe Mix P-003 (a) and P-072 (b) demonstrating germline deletions of the EPCAM, MSH2, and KCNK12 genes.

The patient underwent laparoscopic right hemicolectomy, and histopathological examination revealed moderately differentiated adenocarcinoma with a mucinous component in the ascending colon tumor.
After obtaining the patient's informed consent, we analyzed the patient's tumor and found a high frequency of microsatellite instability. Then, after genetic counseling, we performed genetic analysis using next-generation 


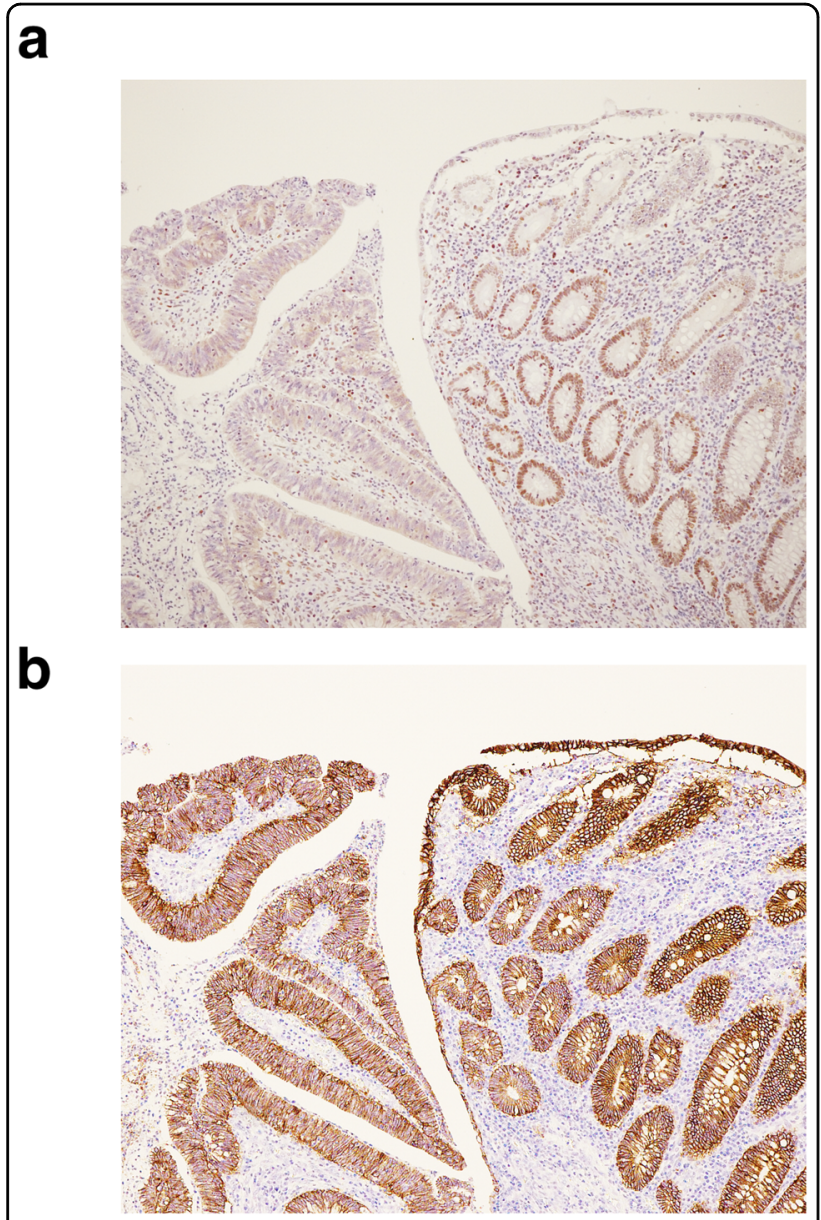

Fig. 3 Immunohistochemistry for mismatch repair protein in ascending colon cancer. Tumor tissue showed loss of staining for MSH2 (a) while staining is retained for EPCAM (b).

sequencing $^{6}$. The assay detected deletion of the EPCAM and $M S H 2$ genes. We conducted further analysis using multiplex ligation-dependent probe amplification (MLPA) to confirm the deletion. Assays were performed to detect large genomic rearrangements of the EPCAM and $M S H 2$ genes using an $M L H 1 / M S H 2$ MLPA kit following the manufacturer's protocol (SALSA MLPA KIT P-003 MLH1/MSH2, MRC-Holland, Amsterdam, The Netherlands). Then, we detected germline deletions of all $M S H 2$ exons and exon 9 of the EPCAM gene. An additional assay was performed using an MSH6 MLPA kit (SALSA MLPA KIT P-072 MSH6), and we detected the deletion of the EPCAM gene and exon 2 of the KCNK12 gene, which was located upstream of the EPCAM gene (Fig. 2a, b).

We identified a novel germline deletion of chromosome 2p16-21, including the EPCAM, MSH2, and KCNK12 genes. Large genomic deletions and duplications, such as this one, have recently been identified using MLPA ${ }^{5}$. However, to our knowledge, there are no reports of germline deletions in this region.
The $M S H 2$ gene is more likely to be found in genomic rearrangements at the locus of this disease because the MSH2 gene has a higher number and density of Alu repeats than other MMR genes ${ }^{7}$. At least $20 \%$ of germline $M S H 2$ pathogenic variants are deletions of exons or multiple exons.

MSH2 variant carriers have a significantly higher risk of developing cancer of the urinary tract compared with carriers of $M L H 1$ and MSH6 mutations ${ }^{8}$. Moreover, male $M S H 2$ variant carriers have a high risk of developing cancer of the stomach ${ }^{9}$. However, our patient and his relatives had not developed urinary tract cancer or stomach cancer.

The difference in cancer risks between EPCAM deletion carriers and $M S H 2$ variant carriers has been reported. The cumulative risk of endometrial cancer in EPCAM deletion carriers is lower than that in $M S H 2$ variant carriers, while the cumulative risk of colorectal cancer in EPCAM deletion carriers is similar to that in $M S H 2$ variant carriers ${ }^{10}$. On the other hand, the cumulative risk of colorectal cancer and endometrial cancer in carriers of a concurrent deletion of the EPCAM and $M S H 2$ genes is reported to be similar to that in $M S H 2$ variant carriers ${ }^{10}$. However, none of our patient's relatives had developed endometrial cancer. In our study, to confirm the role of MMR proteins in carcinogenesis, immunohistochemical staining for $\mathrm{MSH} 2$ and EPCAM was conducted on colorectal cancer tissue, which showed only the loss of MSH2 expression (Fig. 3a, b). Thus, in our patient's colorectal cancer, dysfunction of the MSH2 protein caused dysfunction of the MMR system, followed by a DNA replication error. Therefore, if the cancer risks were different between the $M S H 2$ and EPCAM genes, the expression of MMR proteins should also be considered.

The KCNK12 gene located on 2p22-2p21 encodes one of the subfamilies of two-pore-domain potassium channels ${ }^{11}$. KCNK12 expression has been found in various organs, such as the pancreas, heart, skeletal muscle, ovary, testis, prostate, colon, peripheral blood leukocytes, small intestine, spleen, and thymus ${ }^{11}$. By setting and modulating the cellular membrane potential, potassium channels play a significant role in neuronal activity, muscular excitability, and hormone secretion ${ }^{12}$. Recent evidence supports the hypothesis that alterations in the expression and function of two-pore-domain potassium channels may contribute to cancer development and progression ${ }^{13}$, including breast cancer, colorectal cancer, leukemia, and lymphoma ${ }^{14-16}$. Further, a single-nucleotide polymorphism within the KCNK12 gene has been identified as a candidate genetic marker for migraine in the Finnish population ${ }^{17}$. However, our patient and his relatives did not have migraines or a neurovascular disorder.

The limitations of this report include the absence of segregation analysis and no identification of a breakpoint or rearranged genome. In conclusion, we identified a novel large germline deletion of chromosome 2p16-21, 
including the EPCAM, MSH2, and KCNK12 genes. However, the clinical significance of germline deletion of the KCNK12 gene is still unknown. Therefore, it is necessary to obtain and examine more data on this topic.

\section{HGV database}

The relevant data from this Data Report are hosted at the Human Genome Variation Database at: https://doi.org/10.6084/m9.figshare.hgv.2990; https:// doi.org/10.6084/m9.figshare.hgv.2993; https://doi.org/10.6084/m9.figshare. hgv.2996.

\section{Author details}

'Department of Surgery, Tokyo Metropolitan Cancer and Infectious Diseases Center Komagome Hospital, Tokyo, Japan. ${ }^{2}$ Department of Clinical Genetics, Tokyo Metropolitan Cancer and Infectious Diseases Center Komagome Hospital, Tokyo, Japan. ${ }^{3}$ Diagnosis and Therapeutics of Intractable Diseases and Intractable Disease Research Center, Juntendo University Graduate School of Medicine, Tokyo, Japan. ${ }^{4}$ Department of Pathology, Tokyo Metropolitan Cancer and Infectious Diseases Center Komagome Hospital, Tokyo, Japan. ${ }^{5}$ Department of Digestive Tract and General Surgery, Saitama Medical Center, Saitama Medical University, Kawagoe, Japan

\section{Author contributions}

All authors contributed to this work. S.N. prepared the description of the clinical course and wrote the manuscript; H.E., Y.O., and H.I. performed the genetic analysis; and T.Y. performed surgery, designed the study, and wrote the manuscript.

\section{Conflict of interest}

The authors declare no competing interests.

\section{Publisher's note}

Springer Nature remains neutral with regard to jurisdictional claims in published maps and institutional affiliations.

\section{Received: 10 November 2020 Revised: 9 March 2021 Accepted: 1 April} 2021.

Published online: 19 May 2021

\section{References}

1. Yamaguchi, T. et al. Accumulation profile of frameshift mutations during development and progression of colorectal cancer from patients with hereditary nonpolyposis colorectal cancer. Dis. Colon Rectum 49, 399-406 (2006).
2. Ligtenberg, M. J. L., Kuiper, R. P., van Kessel, A. G. \& Hoogerbrugge, N. EPCAM deletion carriers constitute a unique subgroup of Lynch syndrome patients. Fam. Cancer 12, 169-174 (2013).

3. Niessen, R. C. et al. Germline hypermethylation of MLH1 and EPCAM deletions are a frequent cause of Lynch syndrome. Genes Chromosomes Cancer $\mathbf{4 8}$, 737-744 (2009)

4. Kuiper, R. P. et al. Recurrence and variability of germline EPCAM deletions in Lynch syndrome. Hum. Mutat. 32, 407-414 (2011).

5. Eguchi, $\mathrm{H}$. et al. Identification of a Japanese Lynch syndrome patient with large deletion in the $3^{\prime}$ region of the EPCAM gene. JJCO 46, 178-184 (2016).

6. Kohda, M. et al. Rapid detection of germline mutations for hereditary gastrointestinal polyposis/cancers using HaloPlex target enrichment and highthroughput sequencing technologies. Fam. Cancer 15, 553-562 (2016).

7. Van der Klift, H. et al. Molecular characterization of the spectrum of genomic deletions in the mismatch repair genes MSH2, MLH1, MSH6, and PMS2 responsible for hereditary nonpolyposis colorectal cancer (HNPCC). Genes Chromosomes Cancer 44, 123-138 (2005).

8. Joost, P., Therkildsen, C., Dominguez-Valentin, M., Jonsson, M. \& Nilbert, M. Urinary tract cancer in Lynch syndrome; increased risk in carriers of MSH2 mutations. Urology 86, 1212-1217 (2015).

9. Capelle, L. G. et al. Risk and epidemiological time trends of gastric cancer in Lynch syndrome carriers in the Netherlands. Gastroenterology 138, 487-492 (2010).

10. Kempers, M. J. et al. Risk of colorectal and endometrial cancers in EPCAM deletion-positive Lynch syndrome: a cohort study. Lancet Oncol. 12, 49-55 (2011).

11. Girard, C. et al. Genomic and functional characteristics of novel human pancreatic 2P domain K+ channels. Biochem. Biophys. Res. Commun. 282, 249-256 (2001).

12. Jan, L. Y. \& Jan, Y. N. Potassium channels and their evolving gates. Nature $\mathbf{3 7 1}$ 119-122 (1994).

13. Comes, N. et al. Involvement of potassium channels in the progression of cancer to a more malignant phenotype. Biochem. Biophys. Acta $\mathbf{1 8 4 8}$ 2477-2492 (2015)

14. Keith, A. D., Zhang, W., Stayner, L. \& Argos, M. Associations of two-pore domain potassium channels and triple negative breast cancer subtype in The Cancer Genome Atlas: systematic evaluation of gene expression and methylation. BMC Res. Notes 10, 475 (2017).

15. Kober, P., Bujko, M., Oledzki, J., Tysarowski, A. \& Siedlechi, J. A. Methyl-CpG binding column-based identification of nine genes hypermethylated in colorectal cancer. Mol. Carcinog. 50, 846-856 (2011).

16. Gomes, A. Q. et al. Identification of a panel of ten cell surface protein antigens associated with immunotargeting of leukemias and lymphomas by periphera blood gammadelta T cells. Haematologica 95, 1397-1404 (2010).

17. Nyholt, D. R. et al. A high-density association screen of 155 ion transport genes for involvement with common migraine. Hum. Mol. Genet. 17, 3318-3331 (2008). 